\title{
Frequency dependence of the electrical conductivity and dielectric constants of polycarbonate (Makrofol-E) film under the effects of $y$-radiation
}

\author{
Soad Fares (s.fares) \\ National Center for Radiation Research and Technology (NCRRT), AEA, Nasr City, Egypt; sfares2@yahoo.com
}

Received 20 September 2011; revised 22 October 2011; accepted 2 November 2011.

\begin{abstract}
Irradiation effects of $y$-radiation on the physical and electrical properties of polycarbonate (Makrofol-E) film has been studied to be able to investigate the dielectric response of irradiated polymers for a wide range of fluence and frequency. The dielectric constant $\left(\varepsilon^{\prime}\right)$ The loss tangent $(\tan \delta)$, dielectric loss factor $\left(\varepsilon^{\prime \prime}\right)$, the a.c electrical conductivity $(\sigma)$ and the relaxation time $(\tau)$, were measured in the frequency range from (40) $\mathrm{Hz}$ to (4) $\mathrm{MHz}$. These samples were irradiated by means of $\mathrm{y}$-rays from 10 up to 200 KGy. The change in different properties as a function of absorbed dose was studied. Degradation of the polymers leading to amorphisation was observed by increasing the absorbed $y$ dose. The induced changes in the electrical conductivity due to $\gamma$-rays irradiation of Makrofol-E provide a better method for $y$-dose measurements. A semi-empirical equation was developed to use Makrofol-E as a dielectric dosimeter. Furthermore, Makrofol-E has much greater resistance to radiation damage; the attained results suggested strongly the applicability of Makrofol-E to be used in medical products applications.
\end{abstract}

Keywords: Gamma-Ray; Dose-Response; PC Polycarbonate; Makrofol-E Chain Scission; Cross Linking; a.c Electrical Conductivity; Dielectric Constant and Dielectric Loss

\section{INTRODUCTION}

Polymeric materials are unique because of the range of structural forms that can synthesized and the way in which changes can be made in the structure in local or general way. Polymer composites have steadily gained growing importance during the past decade. Appreciable gain in the knowledge of the structure of such materials has been accomplished through electrical conductivity measurements. Also external parameters, such as the effect of $\gamma$-radiation, play a role in the electrical behavior of such polymer [1].

The irradiation of polymeric materials with ionizing radiation (gamma rays, $\mathrm{X}$ rays, accelerated electrons, ion beams) leads to the formation of very reactive intermediates products (excited states, ions and free radicals), which result in rearrangements and/or formation of new bonds. The effects of these reactions are formation of oxidized products, grafts, scission of main chain (degradation) or cross-linking. Often the two processes (degradation-cross-linking) occur simultaneously, and the outcome of the process is determined by a competition between the reactions $[2,3]$. Oxidation and degradation occur gradually with increasing irradiation dose.

Different polymers have different responses to radiation, which are intrinsically related to the chemical structures of the polymers. Polymers with more hydrogen atoms on side (e.g., polyethylene) tend to cross-link with radiation. Polymers with a methyl group (e.g., polypropylene), disubstitutions (e.g., polymethacrylate) and per-halogen substitutions (e.g., polytetrafluoroethlene) would more likely undergo degradation with radiation. Aromatic polymers with benzene rings either in the main chain or on the side (e.g., polycarbonate) are usually radiation resistant [4]. Polycarbonate (PC) is a wellknown engineering thermoplastic with an excellent balance of optical, physical, mechanical, and processing characteristics [5]. Polycarbonate detectors are used as a particle track detector for neutrons and alpha particles detection [6,7]. Moreover, radiation effects on dielectric properties are of particular interest to science and technology and they have many applications in modern engineering [8-11], and the effect of ion irradiation on the dielectric properties of polymers has been also studied earlier [12-14].

It is well known that irradiation enhance the electrical 
conductivity in insulating polymers. This increase in conductivity is attributing to the amplifications of conjugated structure, i.e. fairly great electron freedom. The irregularity in the polymer chains may also give rise to a hopping mechanism which will enhance the conductivity. Among many dielectric of capacitors P. P, PFT films has greatly reduced the volume of the capacitors because of its large electric strength together with its low dissipation factor $(\tan \delta)$ [15]. The improvement of the dielectric properties has been investigated by high electron irradiation of these polymers [16].

The influence of radiation on the properties and performance of a polymer differs according to whether the material degrades or cross-links and this is in turn depends on specific sensitivities or susceptibilities inherent in the polymer backbone. All materials have been found to break down at very high radiation doses, however, the range of doses under which a given polymer will maintain its desirable properties depends greatly on the chemical structure of the polymers. Indeed, below the destructive level of exposure, radiation treatment can impart many benefits and enhance properties of commercial value [17-20].

Therefore in the present work I investigated experimentally the radiation induced conductivity (RIC) for Makrofol-E film — and by gaining sufficient knowledge about these beneficial radiation, induced effect - which are suitable for use as sensitive $\gamma$-dosimeter. A theoretical model is suggested to estimating the RIC in insulating materials.

\section{EXPERIMENTAL PROCEDURE}

Makrofol-E is a bisphenol-A polycarbonate of chemical composition $\left(\mathrm{C}_{16} \mathrm{H}_{14} \mathrm{O}_{3}\right)$, with an average thickness of $0.275 \mathrm{~mm}$ and the surface area of the sample is $(0.612$ $\mathrm{cm}^{2}$ ). Makrofol-E polycarbonate samples were irradiated with different $\gamma$-doses at room temperature in air with ${ }^{60} \mathrm{Co}$-gamma cell. The applied radiation dose on the Makrofol-E sample was (10 - 200) KGy. Makrofol polycarbonate samples were cut into square pieces and coated with silver paste to achieve ohmic contacts. The measurements of dielectric constant $\left(\varepsilon^{\prime}\right)$, dielectric loss factor $\left(\varepsilon^{\prime \prime}\right)$ and loss tangent $\tan (\delta)$ before and after being irradiated were measured using 3531 ZHITESTER (RLC) digi bridge manufactured by HIOKI, E.E. Corporation, having a frequency range of $(40 \mathrm{~Hz})$ up to $(4 \mathrm{MHz})$ in the room temperature. The dielectric parameters $\left(\varepsilon^{\prime}\right)$ and $\left(\varepsilon^{\prime \prime}\right)$ and the electrical conductivity $(\sigma)$ were evaluate using the conventional formula discussed [21,22]. These formulas are:

$$
\begin{aligned}
& \varepsilon^{\prime \prime}=L c / A \varepsilon_{0} \\
& \tan \delta=1 / R w c
\end{aligned}
$$

$$
\begin{aligned}
& \varepsilon^{\prime \prime}=\varepsilon^{\prime} \tan d \delta \\
& \sigma(\omega)=L / A R
\end{aligned}
$$

where

$\varepsilon_{0}:$ is the Permittivity of free space $\left(=8.85 \times 10^{-12}\right.$ $\mathrm{F} / \mathrm{m}), \omega$ : is the circular frequency $L$ and $A$ : are the thickness and cross-sectional area of the samples respectively.

$\operatorname{Tan}(\delta)$ data were obtained directly from the bridge and from which $\left(\varepsilon^{\prime}\right)$ and $\left(\varepsilon^{\prime \prime}\right)$ were calculated using Eqs.1 and 3. Measurements of $\left(\varepsilon^{\prime \prime}\right)$ and $\left(\varepsilon^{\prime}\right)$ have been measured and calculated, so after obtaining these results, they were applied in Debye, $s$ equation [23]. where

$\varepsilon^{\prime} s:$ is the dielectric constant in static field, $\varepsilon_{\infty}^{\prime}:$ is the dielectric constant at the end of the frequency range (minimum value of $\varepsilon^{\prime}$ ), and $\tau$ : is the relaxation time.

Debye, $s$ equation can be modified to give the dielectric parameters in the form of straight line equation.

$$
\begin{aligned}
\varepsilon^{\prime} & =\varepsilon_{\infty}^{\prime}+\frac{\varepsilon_{s}^{\prime}-\varepsilon_{\infty}^{\prime}}{1+\omega^{2} \tau^{2}} \\
\varepsilon^{\prime \prime} & =\frac{\left(\varepsilon_{s}^{\prime}-\varepsilon_{\infty}^{\prime}\right) \omega c}{1+\omega^{2} \tau^{2}} \\
\log \left(\frac{\varepsilon^{\prime \prime}}{\omega}\right) & =\log \left(\varepsilon^{\prime}-\varepsilon_{\infty}^{\prime}\right)+\log \tau
\end{aligned}
$$

Eq.7 is more useful for higher frequencies [24]. From the intercept of the straight line equation, the relaxation time can be calculated.

\section{RESULTS AND DISCUSSION}

The various assumption made in our discussion can be better appreciated by studying the variations of $\left(\varepsilon^{\prime}\right)$ with frequency for different $\gamma$-doses. These variations for Makrofol-E samples are depicted in Figure 1. Dielectric constants $\left(\varepsilon^{\prime}\right)$ measurement were performed over frequency rang from $(40 \mathrm{~Hz})$ to $(4 \mathrm{MHz})$. Figure 1 shows three responses frequency bands for Makrofol-E samples. At all doses, the samples show that in the $(0.2-1.2 \mathrm{MHz})$ frequency range, the $\left(\varepsilon^{\prime}\right)$ slightly decreases as the frequency increases. In the second band (1.2 - 1.7 MHz), it seems to be $\left(\varepsilon^{\prime}\right)$ sharply increase which is due to the main relaxation process. The third frequency band (1.7 $4 \mathrm{MHz})$ shows that $\left(\varepsilon^{\prime}\right)$ sharply decrease with the frequency, which is attributed due to the dielectric dispersion. As evident from the graph, the dielectric constants remain almost constant up to $1.2 \mathrm{MHz}$ and then decreases at higher frequencies. At lower frequencies the motion of the free charge carriers is constant and thus the dielectric constant is constant. As the frequency increases, the charge carriers migrate through the dielectric and get trapped against a defect site and induce an opposite 


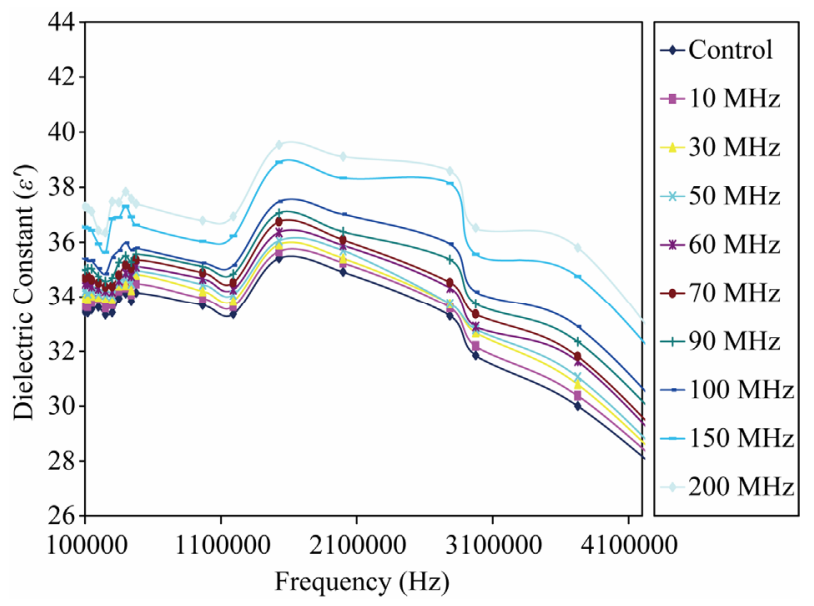

Figure 1. Frequency dependence of the dielectric constant of Makrofol-E samples at different radiation doses.

charge in its vicinity, as a result of which motion of charge carriers is slowed down and the value of dielectric constant decreases.

On the other hand, at all the frequency ranges, significant changes have been observed in dielectric response of Makrofol-E PC after irradiation, the $\left(\varepsilon^{\prime}\right)$ increase by increasing the absorbed dose (10 - $200 \mathrm{KGY})$, which is confirmed in Figure 2. Which shows the dielectric constant versus $\log (f)$. The increasing of $\left(\varepsilon^{\prime}\right)$ as the $\gamma$-doses increase may be attributed due to the oxidative degradation and for the presence of oxygen in air during irradiation.

The variation of dielectric loss factor $\left(\varepsilon^{\prime \prime}\right)$ with frequency at different $\gamma$-doses has been measured. These variations for Makrofol-E samples are depicted in Figure 3. Which shows that more than one note relaxation processes occur. At all, it is interesting to find that, the increase of $\gamma$-dose decreases only the height of these relaxation processes without any shift or effect on the value of the relaxation time. Figure 3 shows the variation of $\left(\varepsilon^{\prime \prime}\right)$ all over the frequency ranges. $\left(\varepsilon^{\prime \prime}\right)$ rapidly decreases in the frequency range $(0.7-1.2) \mathrm{MHz}$ for Makrofol-E sample, $\left(\varepsilon^{\prime \prime}\right)$ increases rapidly in high frequency range with a wide band width, which is attributable to Debye dielectric relaxation, due to a dipole rotations caused by movements of the main backbone.

The variation of $\left(\varepsilon^{\prime \prime}\right)$ all over the frequency ranges shows that more than one relaxation process occurs. The relaxation times data obtained from the analysis using Eq.7 are listed in Table 1. From these calculations, it is notice that, the parameters present of three relaxation regions were defined as they lie within experimental measurements. The three dielectric relaxation regions (which lie approximately at resonance frequencies $f_{c}=$ $0.5 \mathrm{MHz}, f_{c}=1.77 \mathrm{MHz}$ and $f_{c}=2.93 \mathrm{MHz}$ ) are found to be present for all investigated samples. On the other

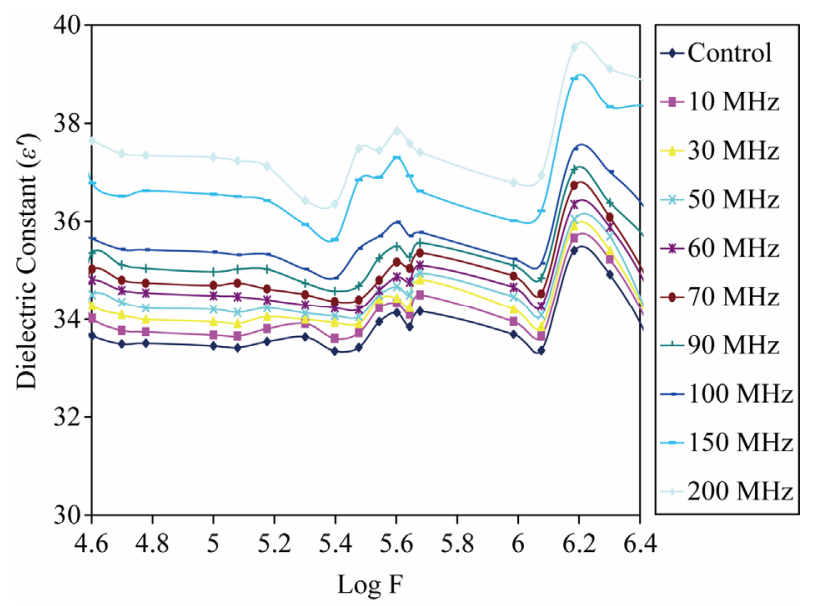

Figure 2. Dielectric constant as a function of Log (Frequency) for different gamma doses Makrofol-E samples.

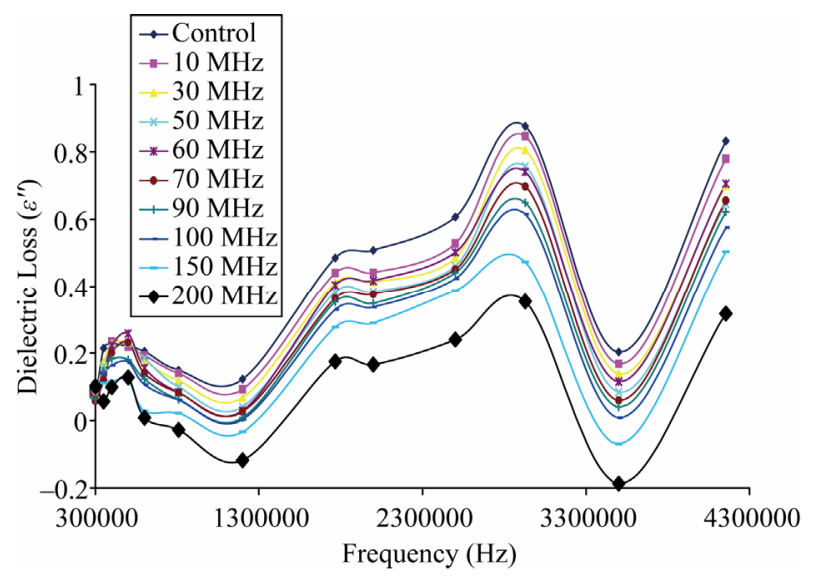

Figure 3. Frequency dependence of the dielectric loss of Makrofol-E samples at different radiation doses.

Table 1. Relaxation data of Macrofol-E film.

\begin{tabular}{ccccc}
\hline $\begin{array}{c}\text { Dielectric } \\
\begin{array}{c}\text { Relaxation } \\
\text { Region }\end{array}\end{array}$ & $f_{c} \mathrm{MHz}$ & $\varepsilon_{1}^{\prime}$ & $\varepsilon_{1}^{\prime \prime}$ & $\tau \mathrm{Sec}$ \\
\hline $1^{\text {st }}$ & 0.5 & 34.15 & 0.215 & $9.5 \times 10^{-9}$ \\
$2^{\text {nd }}$ & 1.77 & 35.14 & 0.485 & $4.7 \times 10^{-9}$ \\
$3^{\text {rd }}$ & 2.93 & 31.84 & 0.875 & $7.9 \times 10^{-9}$ \\
\hline
\end{tabular}

hand, Figure 4 illustrates the plot of $\log \left(\varepsilon^{\prime}-\varepsilon_{\infty}^{\prime}\right)$ versus $\log \left(\varepsilon^{\prime \prime} / \omega\right)$ of the Macrofol-E control sample, from which the value of the mean relaxation time $\tau$ was obtained, from the linear fitting equation, to be $(\tau=2.1 \times$ $10^{-7} \mathrm{sec}$.).

Furthermore, it was noticed a small and activated phenomena at low frequencies $(1750 \mathrm{KHz})$ for Makrofol-E sample, due to local motion of pieces of the polymer backbone in agreement with the observations of Mary. C. Wintersgill et al. [25], while the high peak is due to heat 


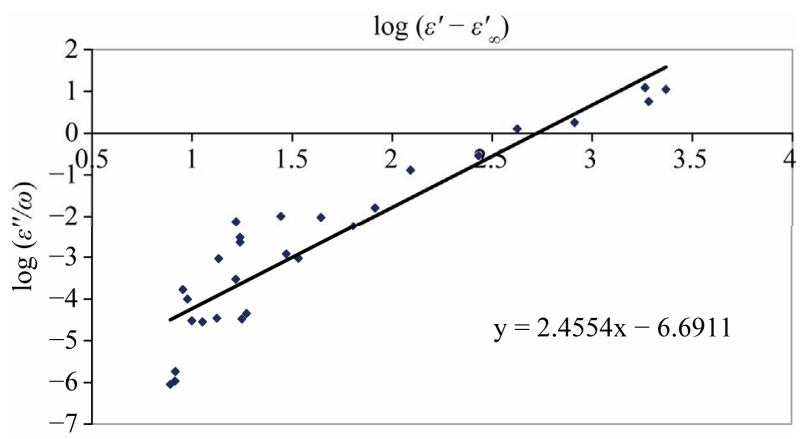

Figure 4. Dielectric relaxation time for the Makrofol-E control sample.

distortion, which appears at $(2.8 \mathrm{MHz})[26,27]$. In all samples, the values of dielectric loss $\left(\varepsilon^{\prime \prime}\right)$ are also found to decrease by increasing the $\gamma$-radiation dose. The dielectric loss is due to the perturbation of the phonon system by an electric field, the energy transferred to the phonons dissipated in the form of heat.

The variation of loss tangent $(\tan \delta)$ with irradiation dose (KGy) is shown in Figure 5. This figure represent the loss behavior at low frequency ranges was found to be appreciable, and no relaxation peaks are observed. Figure 5 shows a liner decrease in $(\tan \delta)$ with $\gamma$-doses for the low frequency rang from $525 \mathrm{KHz}$ to $1.2 \mathrm{MHz}$. From such plots we can use its liners equation, which represented by:

$$
\tan \delta=3.6 \times 10^{-3}-0.22 \times 10^{-4} D
$$

where $(D)$ in KGy

It was observed at low frequency regions mentioned before, as the frequency increases, the $\tan (\delta)$ decreases in these regions (at constant $\gamma$-dose), which means that, these samples cannot be used as dielectric in capacitors.

The variation of the impedance with frequency for different $\gamma$-doses is shown in Figure 6 for the samples under consideration. It can be seen from this curve that the impedance rapidly decrease by increasing frequency until $1.5 \mathrm{MHz}$, above which it remains nearly constant (1.05 MHz) for Makrofol-E sample The variation of a.c. conductivity with frequency was found to obey the conventional formula which discussed before [20]. The dependency of conductivity at high frequency is represented in Figure 7. The increase in conductivity is due to irradiation which attributed to scission of polymer chains and as a result of the absence of dispersion in permittivity of high frequency, suggests that the conduction mechanism is based on electronic hopping, creating energetic free electrons, ions and free radicals [28].

The variation in conductivity with $\gamma$-radiation dose at various frequencies for Makrofol-E samples studied systematically. Figure 8 depict that the change in conductivity, show that a slight increase in conductivity

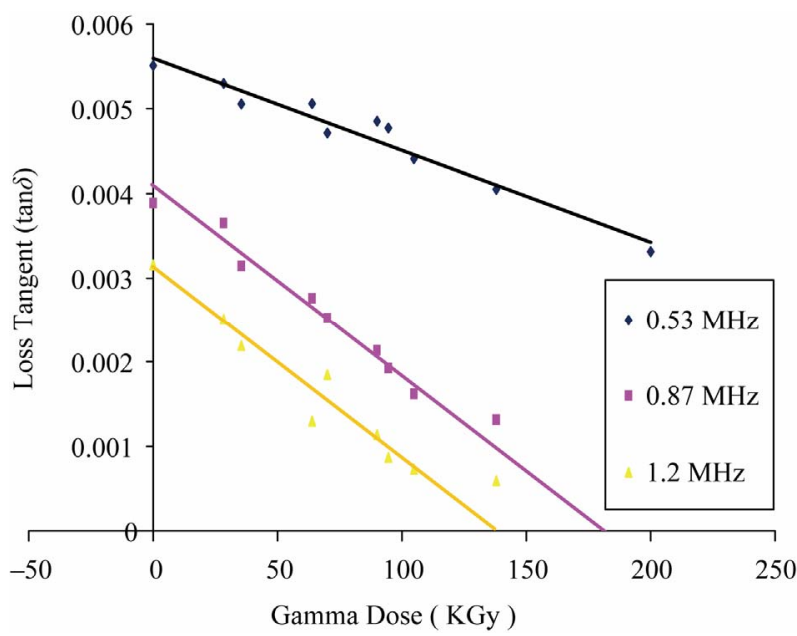

Figure 5. Loss tangent versus gamma dose for Makrofol-E samples at different frequency.

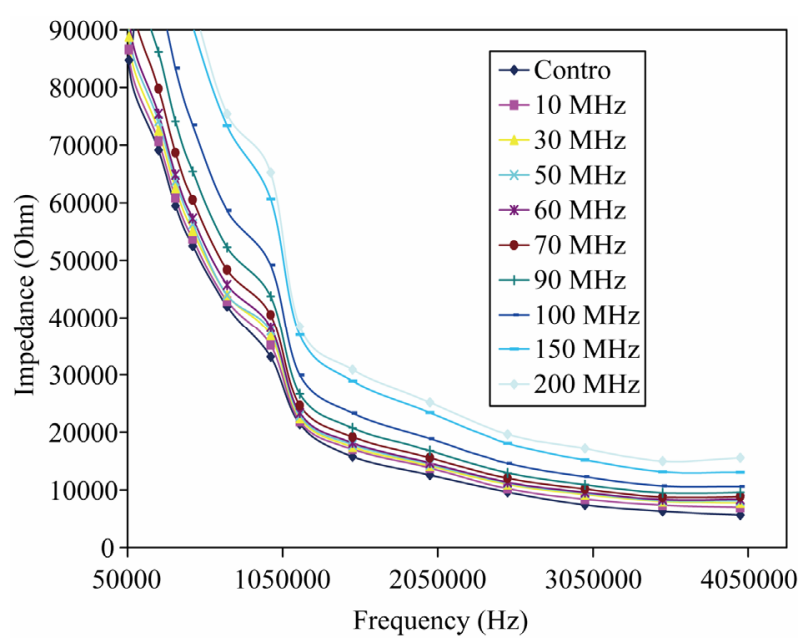

Figure 6. Impedance versus frequency for Makrofol-E samples at different radiation doses.

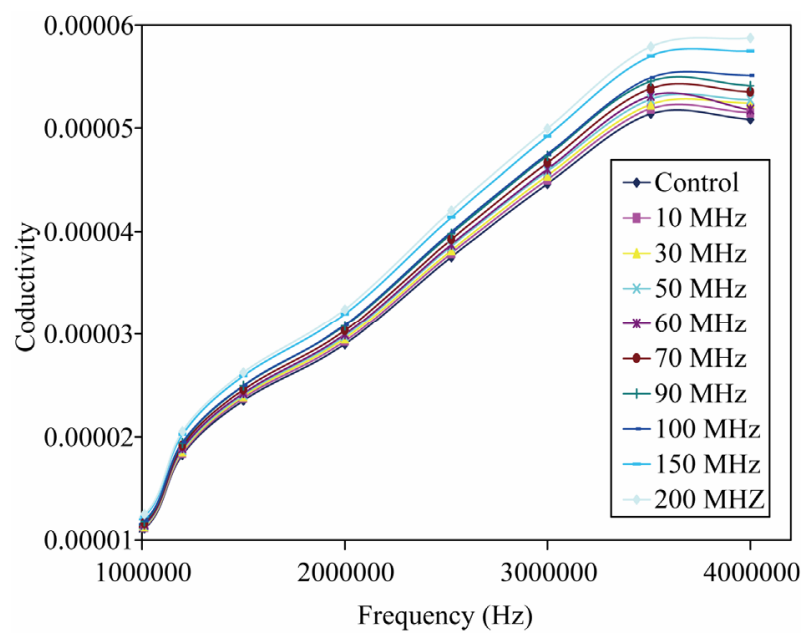

Figure 7. The a.c Conductivity of the Makrofol-E samples versus frequency at different radiation doses. 


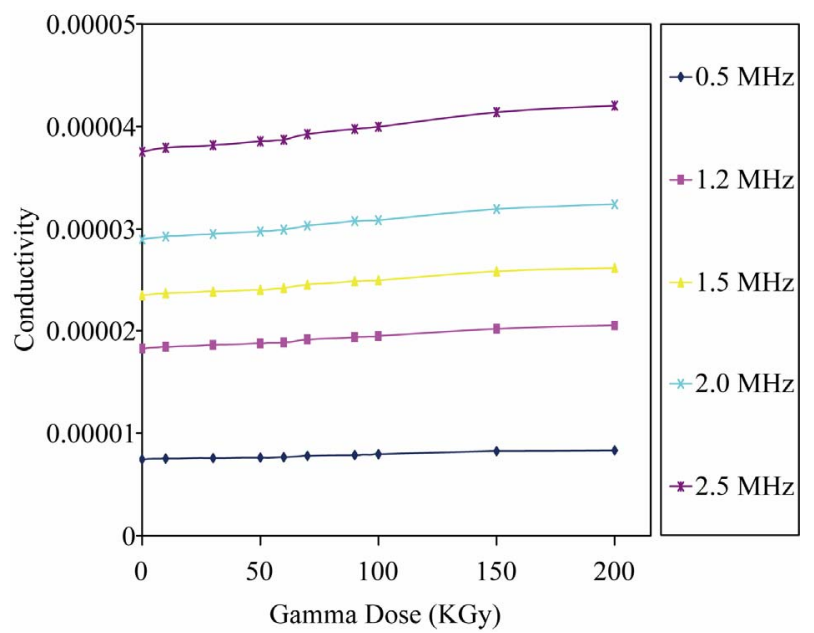

Figure 8. The a.c Conductivity of the Makrofol-E samples versus radiation doses at various frequency values.

with radiation dose. Furthermore, a sharp increase in conductivity was observed within the frequency rang (2 3.5) MHz. The increase in conductivity due to irradiation may be attributed to scissioning of the polymer chains, resulting in an increase of free radicals, unsaturation, etc.

Further more, Figure 8 depicts a linear relation between conductivity and radiation dose up to $200 \mathrm{KGy}$. The empirical equation satisfied by this relation at frequency $(0.6 \mathrm{MHz})$, represented as:

$$
\sigma=7.25 \times 10^{-9} \mathrm{D}+1.2 \times 10^{-5}
$$

where $(D)$ in KGy. Which prove that, the induced changes in electrical conductivity due to gamma irradiation of Makrofol-E provide a better method for gamma dose measurements.

\section{CONCLUSIONS}

Makrofol-E polycarbonate films were irradiated with $\gamma$-radiation to study the modification in dielectric properties induced by irradiation. Ionizing radiation interacts with polymers via two primary mechanisms, chain scission to reduce molecular weight and cross-linking to generate large polymer networks. Both mechanisms occur and its effects vary from polymer to polymers and to some degree from part to part, during irradiation, but one generally dominates. Our experimental results of electrical properties of the Makrofol-E samples prove that, the samples vary in sensitivity to radiation. That is mean, Makrofol-E polymer have much greater resistance to radiation damage, so we suggest Makrofol-E to be used in medical products applications. It has been observed that under $\gamma$-irradiation, dielectric constant decreases with frequency whereas dielectric loss shows random behavior. It increases up to $2.8 \mathrm{MHz}$ followed by a decrease up to $3.5 \mathrm{MHz}$.

\section{ACKNOWLEDGEMENTS}

The auther sincerely thanks prof. Dr.A.korna and Dr. S.M.Abdou, Assi.Prof., for research faciliteis and helpful discussions which enabled this work to be carried out. This work supported by the Departement of Radiation Physics, National Center for Radiation Research and Technology (NCRRT), Cairo, Egypt, is also gratefully acknowledged.

\section{REFERENCES}

[1] Zahran, A.H., Ibrahim, E.M., Ezz-Eldin, F.M. and El-Assy, N.B. (1981) Electrical behaviour of $\gamma$-irradiated rigid and soft poly (vinyl chloride). The International Journal of Applied Radiation and Isotopes, 32, 713-717. doi:10.1016/0020-708X(81)90019-3

[2] Kumar, R., Virk, H.S, Verma, K.C., Saha, A. and Prasad, U.De.R. (2006) Physico-chemical modifications induced in Makrofol N-polycarbonate by swift heavy ions. $\mathrm{Nu}$ clear Instruments and methods in Physics Research B, 251, 163-166. doi:10.1016/j.nimb.2006.06.003

[3] Sinha, D., Sahoo, K.L, Sinha, U.B, Chemseddine, A., Swu, T. and Fink, D. (2004) Gamma induced modifications of polycarbonate polymer. Radiation Effects \& Defects in Solids, 9, 587-595. doi:10.1080/10420150412331330539

[4] Cheng, S. and Kerluke, D.R. (2003) Radiation processing for modification of polymers. 2003 Annual Technical Conference of the Society of Plastic Engineering ( $A N$ TEC) IBA, Advanced Materials Division, 7695 Formula Place, San Diego.

[5] Chung, J.Y.J. (1997) Stabilization of Gamma-Irradiated Polycarbonate. Medical Plastics and Biomaterials. Technical Paper Series, 49.

[6] Buford Price, P. (2008) Recent applications of nuclear tracks in solids. Radiation Measurements, 43, S13-S25. doi:10.1016/j.radmeas.2008.04.002

[7] Nouh, S.A., Morsy, A.A. and EL-Husseniy, H.M. (2004) Radiation-induced changes in some SSNTDs. Radiation Effects \& Defects in Solids, 159, 115-124. doi:10.1080/10420150410001669596

[8] Kecskemeti, G., Smausz, T., Kresz, N., Toth, Z., Hopp, B., Chrisey, D. and Berkesi, O. (2006) Pulsed laser deposition of polyhydroxybutrate biodegradable polymer thin films using ArF excimer laser. Applied Surface Science, 253, 1185-1189. doi:10.1016/j.apsusc.2006.01.084

[9] Basha, A.M., Ahmed, M.A, Marey, H.Kh. and Hanafy, T. A. (1996) Neutron dosimetry measurement. Indian Journal of Physics, 70A, 619-625.

[10] Ahmed, M.A., Basha, A.M., Marey, H.K. and Hanafy, T.A. (2001) Effect of neutrons and $\gamma$ radiation on cobalt-gelatin Film. Journal of Applied Polymer Science, 79, $1749-1755$.

doi:10.1002/1097-4628(20010307)79:10<1749::AID-AP P20>3.0.CO;2-H

[11] Hanafy, T.A., (2008) Drastic effect of fast neutrons and $\gamma$ radiation on the dc-conductivity of $\mathrm{Co}_{-}, \mathrm{Ni}-, \mathrm{Mn}-$, and Aggelatin doped film. Current Applied Physics, 8, 527534. doi:10.1016/j.cap.2007.09.002

[12] Boiteuxa, G., Chailan, J.F., Chaucharda, J. and Seytrea, G. (1997) Dielectric and mechanical spectroscopes for the study of thermal and radiochemical ageing of poly- 
mers. Dielectric and mechanical spectroscopes for the study of thermal and radiochemical ageing of polymers, 131, 172-179. doi:10.1016/S0168-583X(97)00198-5

[13] Esther Martínez-Pardo, Ma., Cardoso, J., Vázquez, H. and Aguilar, M. (1998) Characterization of MeV proton irradiated PS and LDPE thin films. Nuclear Instruments and Methods in Physics Research Section B, 140, 325340. doi:10.1016/S0168-583X(98)00013-5

[14] Phukan, T., Kanjilal, D., Goswami, T. D. and Beam, H. L. (1999) Interactions with Materials and Atoms. Nuclear Instruments and Methods in Physics Research Section B, 155, 116-119. doi:10.1016/S0168-583X(99)00255-4

[15] Yoshida, Y., Nishimatsu, N., Mukai, S., Kashiwazaki, T. and Yasufuku, S. (1980) Evolution of Power Capacitors as a Result of New Material Development. CIGRE Report, 15-01.

[16] Kita, H., Okamoto, K. and Sakamoto, I. (1986) Physical and dielectric properties of irradiated polypropylene and poly (ethylene terephthalate). International Journal of Radiation Applications and Instrumentation. Part C. Radiation Physics and Chemistry, 28, 393-397.

[17] Information Sources Include Polymer Manufacturers Data (1988) NASA/Jet Propulsion Laboratories.

[18] Skeins and Williams (1992) "Ionizing Radiation Effect on Selected Biomedical Polymers"; Kiang, "Effect of Gamma Irradiation on Elastomeric Closures," Technical Report, 16, PDA.

[19] Kumar, R., Asad Ali, S., De, U., Avasthi, D. K. and Prasad, R. (2009) Dielectric response of makrofol-KG polycarbonate irradiated with $145 \mathrm{MeV} \mathrm{Ne}{ }^{6+}$ and 100 $\mathrm{MeV} \mathrm{Si}^{8+}$ ions. Indian Journal of Physics, 83, 963-968. doi:10.1007/s12648-009-0055-6

[20] Abul-Hail, R.Ch. (2010) Optical absorption of polycar- bonate (Makrofol E) as means of Gamma-ray Dosimetry. Journal of Basrah Researches (Sciences), 36, 26-30.

[21] Elliott, S.R. (1087) A.c. conduction in amorphous chalcogenide and pnictide semiconductors. Advances in Physics, 36, 135-217. doi:10.1080/00018738700101971

[22] Bhatnagar, V.K. and Bhatia, K.L. (1990) Frequency dependent electrical transport in bismuth-modified amorphous germanium sulfide semiconductors. Journal of Non-Crystalline Solids, 119, 214-231. doi:10.1016/0022-3093(90)90845-D

[23] Fawzy, Y.H. (2002) Study on spectroscopic determination and electrical properties of rare earth minerals. Ph.D Thesis, Faculty of Girls, Ain Shams University, Cairo, Egypt.

[24] Pethig, R. (1979) Dielectric and electronic properties of biological materials. Wiley, New York, 15-18.

[25] Wintersgill, M.C. and Fontanella, J.J. (1998) Complex impedance measurements on Nafion. Electrochimica Acta, 43, 1533-1538. doi:10.1016/S0013-4686(97)10049-4

[26] El-Sayed, A.H., Abd El-Rehim, H.A., Khalifa, N.A. and El-Hag Ali, A. (1999) Preparation and characterization of supported hydrogels obtained by radiation grafting of binary monomers. Radiation Physics and Chemistry, 55, 219-229. doi:10.1016/S0969-806X(98)00329-6

[27] Abdou, S.M. and Mohamed, R.I. (2002) Characterization of structural modifications in poly-tetra-fluoroethylene induced by electron beam irradiation. Journal of Physics and Chemistry of Solids, 63, 393-398.

[28] Czvikovszky, T. and Hargitai, H. (1999) Compatibilization of recycled polymers through radiation treatment. Radiation Physics and Chemistry, 55, 727-730. doi:10.1016/S0969-806X(99)00220-0 